\title{
One-pot production of butyl butyrate from glucose using a cognate "diamond-shaped" E. coli consortium
}

\author{
Jean Paul Sinumvayo ${ }^{1,2}$, Chunhua Zhao ${ }^{1,2}$, Guoxia Liu' ${ }^{1}$, Yin Li ${ }^{1^{*}}$ and Yanping Zhang ${ }^{1 *}$
}

\begin{abstract}
Esters are widely used in plastics, textile fibers, and general petrochemicals. Usually, esters are produced via chemical synthesis or enzymatic processes from the corresponding alcohols and acids. However, the fermentative production of esters from alcohols and/or acids has recently also become feasible. Here we report a cognate microbial consortium capable of producing butyl butyrate. This microbial consortium consists of two engineered butyrate- and butanol-producing E. coli strains with nearly identical genetic background. The pathways for the synthesis of butyrate and butanol from butyryl-CoA in the respective $E$. coli strains, together with a lipase-catalyzed esterification reaction, created a "diamond-shaped" consortium. The concentration of butyrate and butanol in the fermentation vessel could be altered by adjusting the inoculation ratios of each E. coli strain in the consortium. After optimization, the consortium produced $7.2 \mathrm{~g} / \mathrm{L}$ butyl butyrate with a yield of $0.12 \mathrm{~g} / \mathrm{g}$ glucose without the exogenous addition of butanol or butyrate. To our best knowledge, this is the highest titer and yield of butyl butyrate produced by E. coli reported to date. This study thus provides a new way for the biotechnological production of esters.
\end{abstract}

Keywords: Butyl butyrate, E. coli, Cognate consortium, Butanol, Butyrate

\section{Introduction}

Fatty acid esters are a large group of value-added chemicals derived from short-chains alcohols and carboxylic acids. They are present in natural sources such as flowers, fermented beverages, and particularly in fruits (Chung et al. 2015; Jenkins et al. 2013). Notably, butyl butyrate (BB) is known as a flavor and fragrance compound that is widely used in foods, beverages, perfumes, and cosmetics (Santos et al. 2007). BB is also an important solvent widely used in the production of plastics, fibers, and processing of petroleum products (Horton and Bennett 2006; Matte et al. 2016).

Like most esters (R1COOR2), BB is traditionally produced by esterification of butyrate and butanol, which is

\footnotetext{
*Correspondence: yli@im.ac.cn; zhangyp@im.ac.cn

${ }^{1}$ CAS Key Laboratory of Microbial Physiological and Metabolic Engineering, State Key Laboratory of Microbial Resources, Institute of Microbiology, Chinese Academy of Sciences, Beijing 100101, China Full list of author information is available at the end of the article
}

usually conducted using inorganic catalysts at relatively high temperatures (Ju et al. 2011; Kang et al. 2011), but an enzymatic process for the production of BB has also been developed (Van den Berg et al. 2013; Matte et al. 2016). While current catalytic and enzymatic BB production processes all require the external supplementation of butanol and butyrate, some Clostridium species are able to produce butyrate, and can further convert the produced butyrate into butanol. However, most of the butyrate produced during acetone-butanol-ethanol fermentation is converted into butanol, leaving insufficient butyrate available for the esterification reaction. Therefore, butyrate, butanol, or both, need to be added to maintain sufficient levels of precursors (Xin et al. 2019). For example, $7.9 \mathrm{~g} / \mathrm{L}$ butyrate had to be supplemented to a fed-batch fermentation of xylose by Clostridium sp. strain BOH3 to produce 22.4 g/L BB (Xin et al. 2016), while $10 \mathrm{~g} / \mathrm{L}$ butanol needed to be added to a fermentation of Clostridium tyrobutyricum to achieve a BB titer of $34.7 \mathrm{~g} / \mathrm{L}$ (Zhang et al. 2017). 
Recently, Cui et al. developed a clostridial consortium comprising the butanol-producing $C$. beijerinckii and the butyrate-producing $C$. tyrobutyricum. They demonstrated that this consortium could produce $5.1 \mathrm{~g} / \mathrm{L} \mathrm{BB}$ without the addition of exogenous substrates. However, the yield of $\mathrm{BB}(0.068 \mathrm{~g} / \mathrm{g})$ was rather low, most likely due to the imbalanced ratio of butanol and butyrate, as well as the production of byproducts such as acetone and isopropanol (Cui et al. 2020). The imbalanced ratio of butanol and butyrate may be exacerbated by the two different species in the clostridial co-culture, with disparate optimal growth conditions. To solve this, we proposed a cognate microbial consortium for BB production, comprising butyrate- and butanol-producing E. coli strains with the same genetic background. If such a pair of cognate $E$. coli strains could be developed, their nearly identical genetic background would allow them to achieve a balanced production of butyrate and butanol by simply adjusting the composition of each strain in the consortium, thus more efficient BB production.

Previously, we developed the chromosomally engineered E. coli strain EB243 capable of efficiently producing butanol from glucose (Dong et al. 2017). We intended to construct another butyrate-producing $E$. coli strain by redirecting the carbon flow at the node of butyl-CoA, thus shifting the carbon flow from butanol to butyrate production. When both strains were co-cultured and supplied with lipase, an E. coli consortium capable of directly producing BB from glucose can be constructed (Fig. 1). In this consortium, the two engineered E. coli strains share the same upstream metabolism, which diverges at the butyryl-CoA node and re-converges at BB, thus forming a "diamond-shaped" consortium (Fig. 1). We demonstrate the feasibility of using such a homogeneous microbial consortium for the production of esters with the assistance of exogenously added lipase in a two-liquid-phase fermentation system, providing a new approach for the biotechnological production of esters.

\section{Materials and methods}

\section{Strains, plasmids and primers}

E. coli EB243 (Dong et al. 2017) was used as the starting strain for metabolic engineering. All strains and plasmids used in this study are listed in Table 1 . All primers (Additional file 1: Table S1) were synthesized by Invitrogen (Beijing, China) and purified via polyacrylamide gel electrophoresis. Candidate genes encoding acyl-CoA thioesterase ( $y$ ciA, tesB) were amplified by PCR from the genomic DNA of E. coli BW25113, while those encoding phosphate butyryltransferase $(p t b)$ and butyrate kinase (buk and buk2) were amplified from the genome of Clostridium acetobutylicum DSM 1731.

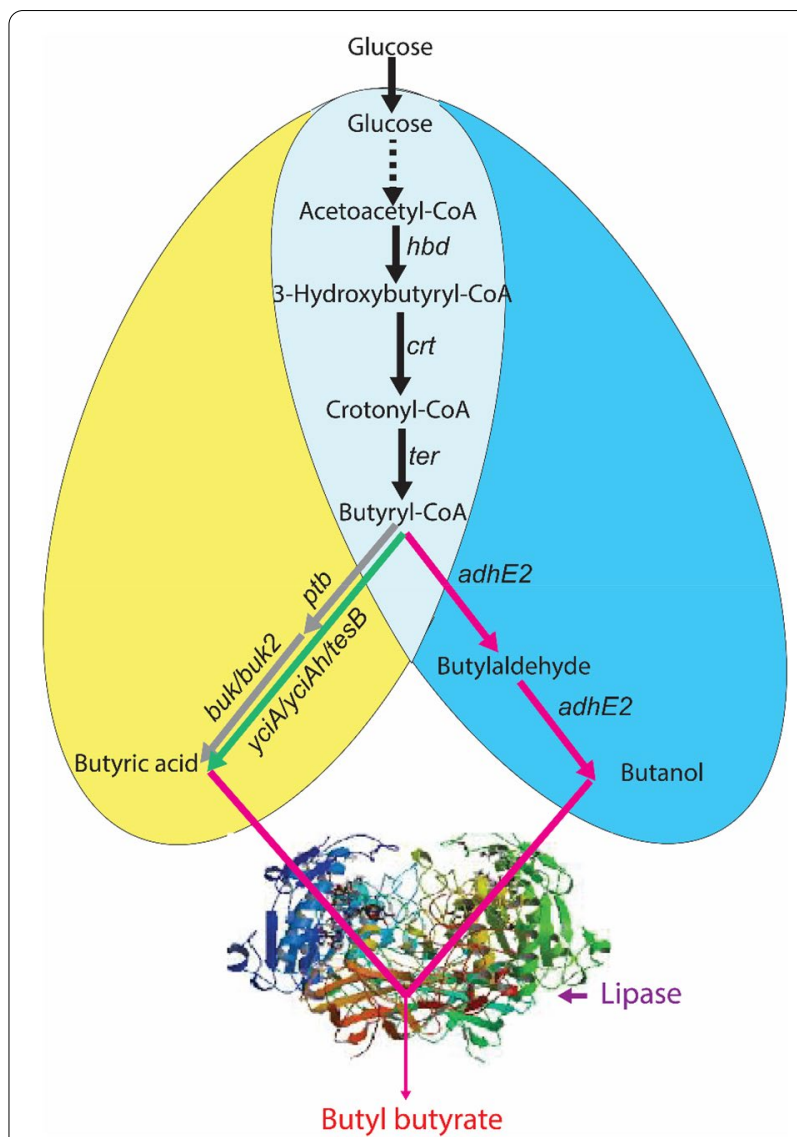

Fig. 1 Construction of a "diamond-shaped" consortium for the direct production of butyl butyrate from glucose using two E. coli strains with the same genetic background. The butyric acid-producing strain is shown in yellow, and the butanol-producing strain in blue. A shared pathway from glucose to butyryl-CoA is shown in light grey in overlap between the two strains. A green arrow indicates the thioesterase pathway (encoded by yciA, yciAh or tesB), while the ptb-buk and ptb-buk2 route is indicated by a grey arrow. The production routes of precursors (butanol and butyric acid) and their conversion into butyl butyrate with the assistance of lipase enzyme forms a "diamond-shaped" structure, which was used to define the consortium. The genes hbd (3-hydroxybutyryl-CoA dehydrogenase), crt (crotonase), adhE2 (aldehyde/alcohol dehydrogenase), ptb (phosphate butyryltransferase), as well as buk and buk2 (butyrate kinase) are derived from C. acetobutylicum; the ter gene (trans-enoyl-CoA reductase) is from Treponema denticola; yciA and tesB (acyl-CoA thioesterase) are from E. coli; yciAh (acyl-CoA thioesterase) is from Haemophilus influenza

The codon-optimized $y c i A h$ variant of the acyl-CoA thioesterase gene yciA from Haemophilus influenzae (Menon et al. 2015) was synthesized by GenScript (Nanjing, China). Subsequently, each gene was cloned into the pAC2 plasmid under the control of the miniPtac promoter (Zhao et al. 2019) and independently expressed in strain EB243 $\triangle$ adhE2, resulting in the plasmids and strains summarized in Table 1. 
Table 1 Strains and plasmids used in this study

\begin{tabular}{|c|c|c|}
\hline Strain or plasmid & Relevant characteristics & Reference or source \\
\hline \multicolumn{3}{|l|}{ Strains } \\
\hline E. coli EB243 & $\begin{array}{l}\text { Derived from BW25113; Containing butanol synthesis pathway genes (atoB, hbd, crt, ter, } \\
\text { adhE2) and fdh, as well as deletions of adhE, eutE, yahD, ackA, pta, hyc-hyp, fdhF, poxB, } \\
\text { pck, fumB, fumAC, tdcD, mdh, focA, ppc, mgsA, yieP, stpA, ygeG, and yagM }\end{array}$ & (Dong et al. 2017) \\
\hline 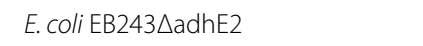 & EB243 derivative, with adhE2 deleted & This study \\
\hline E. coli EB243 $\triangle$ adhE2-pAC2 & EB243 derivative, harboring the plasmid pAC2 & This study \\
\hline E. coli EB243 $\triangle$ adhE2-pAC2-ptb-buk & EB243 derivative, harboring the plasmid pAC2-ptb-buk & This study \\
\hline $\begin{array}{l}\text { E. coli EB243 } \triangle \text { adhE2-pAC2-ptb- } \\
\text { buk2 }\end{array}$ & EB243 derivative, harboring the plasmid pAC2-ptb-buk2 & This study \\
\hline 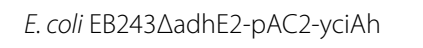 & EB243 derivative, harboring the plasmidpAC2-yciAh & This study \\
\hline 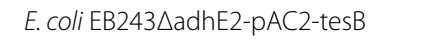 & EB243 derivative, harboring the plasmid pAC2-tesB & This study \\
\hline E. coli EB243 $\triangle$ adhE2-pAC2-yciA & EB243 derivative, harboring the plasmid pAC2-yciA & This study \\
\hline 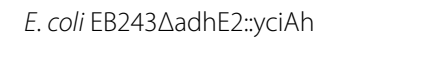 & $\begin{array}{l}\text { Derived from EB243 } \triangle \mathrm{adhE} 2 \text {; with the synthetic thioesterase gene "yciAh" integrated into } \\
\text { the chromosome }\end{array}$ & This study \\
\hline \multicolumn{3}{|l|}{ Plasmids } \\
\hline pAC2 & pACYC184 derivative, miniPtac, cat, $\operatorname{Kan}^{R}$ & (Zhao et al. 2019) \\
\hline pAC2-ptb-buk & pAC2 derivative, expressing the $p t b-b u k$ genes & This study \\
\hline pAC2-ptb-buk2 & pAC2 derivative, expressing the $p t b-b u k 2$ genes & This study \\
\hline pAC2-yciAh & pAC2 derivative, expressing the yciAh gene & This study \\
\hline pAC2-yciA & pAC2 derivative, expressing the yciA gene & This study \\
\hline pAC2-tesB & PAC2 derivative, expressing the tes $B$ gene & This study \\
\hline pTargetF & aadA, guide RNA transcription & (Jiang et al. 2015) \\
\hline pCas & $\mathrm{Kan}^{\mathrm{R}}$, gam-bet-exo, cas 9 & (Jiang et al. 2015) \\
\hline pTargetF-adhE2 & Derived from pTargetF, adhE2 knockout vector & This study \\
\hline
\end{tabular}

cat: chloramphenicol acetyl transferase gene; aadA: spectinomycin resistance gene; Kan ${ }^{\mathrm{R}}$ : kanamycin resistant strain; gam-bet-exo: Red recombinase genes; cas9: Cas9 protein coding gene

\section{Cell culture and fermentation conditions}

For genetic modification, E. coli strains were grown aerobically at $37{ }^{\circ} \mathrm{C}$ in Luria-Bertani (LB) medium $(10 \mathrm{~g} / \mathrm{L}$ tryptone, $5 \mathrm{~g} / \mathrm{L}$ yeast extract, $10 \mathrm{~g} / \mathrm{L} \mathrm{NaCl})$ supplemented with kanamycin $(50 \mu \mathrm{g} / \mathrm{mL})$ when necessary. The strains were preserved in $15 \%$ glycerol at $-80{ }^{\circ} \mathrm{C}$. The cryopreserved cells were first grown overnight on LB plates, after which fresh colonies were picked and used directly to inoculate LB medium, followed by overnight culture at $37^{\circ} \mathrm{C}$ at $200 \mathrm{rpm}$.

Tube fermentation was performed in a sealed 10 or $50 \mathrm{~mL}$ polypropylene conical tube (BD Biosciences, San Jose, CA) containing various volumes of the medium, which was slightly modified from M9Y medium (Dong et al. 2017) (M9 medium $+2 \mathrm{~g} / \mathrm{L}$ yeast extract $+20 \mathrm{~g} / \mathrm{L}$ glucose). Cells were cultured at $37{ }^{\circ} \mathrm{C}$ with constant shaking at $200 \mathrm{rpm}$ for $48 \mathrm{~h}$ or longer where indicated. Samples comprising $0.5 \mathrm{~mL}$ of the fermentation broth were harvested every $24 \mathrm{~h}$, and centrifuged at $19,216 \times g$ for $1 \mathrm{~min}$. The supernatant was filtered through a $0.22 \mu \mathrm{m}$ pore-size filter membrane (nylon), and transferred into $2 \mathrm{~mL}$ HPLC vials for analysis of residual sugar and metabolites.

Fermentations were conducted in $1 \mathrm{~L}$ Infors HT bioreactors containing $0.9 \mathrm{~L}$ fermentation medium (M9 medium $+5 \mathrm{~g} / \mathrm{L}$ yeast extract $+60 \mathrm{~g} / \mathrm{L}$ glucose) with air sparging. The agitation speed was set to $200 \mathrm{rpm}$ and the $\mathrm{pH}$ was maintained at 6.8 by the automatic addition of $5 \mathrm{M}$ $\mathrm{NaOH}$. During fermentation process, $1 \mathrm{~mL}$ of the fermentation broth was withdrawn for analysis every $24 \mathrm{~h}, 0.5 \mathrm{~mL}$ of which was used for cell growth monitoring, while the other $0.5 \mathrm{~mL}$ was used for HPLC as described above.

The final $\mathrm{OD}_{600}$ of the butanol- and butyrate-producing strains after overnight culture in LB medium were not the same but they did not differ much. The two cultures were inoculated in a designed OD ratio. To calculate the precise volume of butanol- and butyrate-producing strains to be inoculated to achieve a designed inoculum ratio, we used the system of equations:

$$
\text { Ratio of } \frac{\text { Butyrate strain }}{\text { Butanol strain }}=\frac{X V 1}{Y V 2}
$$




$$
a=X V 1+Y V 2
$$

where $X$ represents the OD of the butanol-producing strain, $Y$ represents the OD of the butyrate-producing strain, while $V 1$ and $V 2$ represent the respective volumes of the butanol- and butyrate-producing strains to be inoculated.

The starting optical density for inoculation was set at 0.2 and 0.4 for tube and bioreactor fermentation, respectively. Therefore, the value of $a$ in Eq. (2) is the total OD to be inoculated, which can be $1,2,3,4,6$, or 400 when the fermentation broth is $5,10,15,20,30 \mathrm{~mL}$ in tubes, or $1000 \mathrm{~mL}$ in the bioreactor, respectively. For example, when using a ratio of 1:4 in a bioreactor experiment with $1000 \mathrm{~mL}$ of fermentation broth, the initial inoculum volume of the two strains was determined using the following system of equations:

$$
\begin{aligned}
& \frac{1}{4}=\frac{X V 1}{Y V 2} \\
& 400=X V 1+Y V 2
\end{aligned}
$$

\section{Production of $B B$ from glucose}

For $\mathrm{BB}$ production, overnight cultures of the butyrateproducing strain EB243AadhE2::yciAh and butanolproducing strain EB243 (at an inoculation ratio of 1:4), were seeded into $15 \mathrm{~mL}$ M9Y medium in a sealed $50 \mathrm{~mL}$ conical polypropylene tube. Additionally, $5 \mathrm{~g} / \mathrm{L}$ of LCS (recombinant lipase from Candida sp., expressed in Aspergillus niger; Novozymes Lipozyme ${ }^{\circledR}$ CALB, SigmaAldrich) was used to convert the butanol and butyrate into BB. CALB should be added when the substrate is available. The concentrations of butyrate and butanol after 8,10 , and $12 \mathrm{~h}$ of fermentation were then determined. To extract the produced $\mathrm{BB}, 15 \mathrm{~mL}$ hexadecane (Sigma-Aldrich) was added to each $50 \mathrm{~mL}$ conical polypropylene tube. The cultures were incubated in a rotary shaking incubator at $200 \mathrm{rpm}$ and $37^{\circ} \mathrm{C}$ for $72 \mathrm{~h}$. Every $24 \mathrm{~h}, 1 \mathrm{~mL}$ of culture was collected for analysis of metabolites and residual sugar. At the same time, $1 \mathrm{~mL}$ of the hexadecane layer was also sampled to detect the concentration of the produced $\mathrm{BB}$. The produced $\mathrm{BB}$ is efficiently extracted into the organic phase, as a previous study demonstrated that the partitioning coefficient for $\mathrm{BB}$ in the hexadecane/aqueous system is more than 300 (Zhang et al. 2017). To ensure the detection of all $\mathrm{BB}$, the concentration of butyl butyrate in the aqueous phase was also measured, but BB could not be detected and its aqueous solubility can thus be neglected. All data on the concentration of $\mathrm{BB}$ therefore refer to what was detected in the organic phase. Since the volume of the organic phase and aqueous culture broth was $1: 1$, the concentration of $\mathrm{BB}$ in the organic phase was equal to the concentration produced in the aqueous culture broth.

\section{Genetic manipulation and strain development}

The simultaneous knockout of $a d h E 2$ and the integration of $y c i A h$ with a strong RBS and miniPtac promoter in the chromosome of strain EB243 was carried out using a published CRISPR/Cas9 (Jiang et al. 2015). Briefly, a pTargetF-derivative plasmid harboring a designed N20 DNA sequence from the genomic target gene and the corresponding homologous fragment was used to cotransforme fresh $E$. coli competent cells along with the pCas plasmid, which expresses Cas9 protein and Red recombinase. The correct transformants were screened by colony PCR and confirmed by DNA sequencing. Inducing pCas with IPTG results in cells free from the pTargetF vector, while pCas can be cured by cultivating mutant cells at an elevated temperature since pCas is temperature-sensitive. For $y c i A h$ integration, the primers pTargetF-adhE2N20-1/pTargetF-2 were used to amplify pTargetF-adhE2 containing the designed N20 sequence, while the primer pairs adhE2-up-F/adhE2-up- $R$ and adhE2-down-F/adhE2-down- $R$ were used to amplify the homologous arms. Similarly, yciAh-F with adhE2-up-R half homologous sequence and yciAh- $R$ with adhE2down-F half homologous sequence was used to amplify the $y c i A h$ gene for chromosomal integration. Then, the three fragments were fused to form the homologous sequence. Subsequently, pTargetF-adhE2, and the homologous sequence were introduced into the EB243 strain harboring pCas. The resulting mutant strain was verified by colony PCR using the primer pair adhE2-up-F/ adhE2-down-R. In the end, we obtained a plasmid-free strain by applying the curing strain strategy as described above. The integrated sequences were amplified from the constructed pAC2-based plasmids (Table 1), constructed using the Gibson assembly kit (New England BioLabs, Beijing, China).

\section{Analytical methods}

To assess strain growth, the optical density at $600 \mathrm{~nm}$ $\left(\mathrm{OD}_{600}\right)$ was measured using a UV-2802PC; spectrophotometer (Unico, Shanghai, China). The concentrations of butyrate, butanol, and glucose in the fermentation samples were measured by HPLC using an Agilent 1260 system (Agilent Technologies, Santa Clara, CA, USA), equipped with an HPX-87H column (Bio-Rad Laboratories, Inc., Richmond, CA, USA) kept at $55^{\circ} \mathrm{C}$, with $5 \mathrm{mM}$ $\mathrm{H}_{2} \mathrm{SO}_{4}$ at a flow rate of $0.5 \mathrm{~mL} / \mathrm{min}$ as the mobile phase. The injection volume was $10 \mu \mathrm{L}$ injection. For measurement of BB production, samples were taken from the solvent phase during fermentation, filtered, and immediately 
analyzed on a GCMS-QP2010 Ultra (Shimadzu, Japan) system equipped with a DB-5 ms column (30 m length, $0.25 \mathrm{~mm}$ inside diameter, $0.25 \mu \mathrm{m}$ thickness, Agilent, USA). The flow rate of the helium carrier gas was $1 \mathrm{~mL} /$ $\mathrm{min}$. The interface and ion source temperatures were set to 250 and $200{ }^{\circ} \mathrm{C}$, respectively. The electron impact voltage was set to $70 \mathrm{eV}$. The $m / z$ range was $35-500$. The column temperature was initially set to $100{ }^{\circ} \mathrm{C}$, after which it was increased to $250{ }^{\circ} \mathrm{C}$ at a rate of $20^{\circ} \mathrm{C} / \mathrm{min}$, where it was held for $5 \mathrm{~min}$.

\section{Statistical analysis}

Statistical analysis using Student's $t$-test and plotting of diagrams was performed in Origin software. $P$ values of $<0.05$ were considered to indicate statistical significance.

\section{Results and discussion}

\section{Construction of a butyrate-producing strain}

A butyrate-producing strain was constructed starting from the chromosomally engineered $E$. coli strain EB243, which is capable of efficiently producing butanol from glucose (Dong et al. 2017). Since the production of butyrate and butanol diverges at the node of butyryl$\mathrm{CoA}$, a straightforward engineering strategy would be to block the butanol synthesis of strain EB243 while simultaneously introducing a suitable enzyme capable of converting butyryl-CoA into butyrate. Thioesterase, butyrate kinase, and phosphate butyryltransferase are all capable of catalyzing this reaction. Therefore, three acyl-CoA thioesterase genes ( $y c i A$ and tesB from $E$. coli, as well as $y c i A h$ from $H$. influenzae), one phosphate butyryltransferase gene ( $p t b$ from $C$. acetobutylicum), and two butyrate kinases genes (buk and buk2 from $C$. acetobutylicum) were selected for testing.

The starting strain E. coli EB243 (Dong et al. 2017) only generated $0.25 \mathrm{~g} / \mathrm{L}$ butyrate after $72 \mathrm{~h}$ of fermentation, suggesting a very weak butyrate production ability. This is due to the string activity of aldehyde/alcohol dehydrogenase (AdhE2), which was introduced for butanol production. Therefore, adhE2 had to be deleted and genes responsible for butyrate formation had to be introduced. Accordingly, adhE2 was first deleted to form the strain EB243AadhE2. Subsequently, the aforementioned selected genes from various sources were expressed to increase the titer and yield of butyrate. Plasmids harboring the genes of interest were first constructed in $E$. coli DH $5 \alpha$, screened by colony PCR, and verified by sequencing before transformation of the EB243 $\Delta$ adhE2 strain.

\section{Production of butyrate in tube and bioreactor fermentation}

To test if the candidate genes can increase butyrate production, three single genes $(y c i A$, tes $B$, and $y c i A h)$ encoding thioesterase and two gene pairs ( $p t b-b u k$, and $p t b-b u k 2)$, respectively encoding phosphate butyryltransferase and butyrate kinase, were cloned into the pAC2 plasmid and expressed in strain EB243 $\triangle$ adhE2. The resulted strains were individually cultured in $5 \mathrm{~mL}$ M9Y medium in $10 \mathrm{~mL}$ polypropylene centrifuge tubes with appropriate antibiotics. The strain containing the
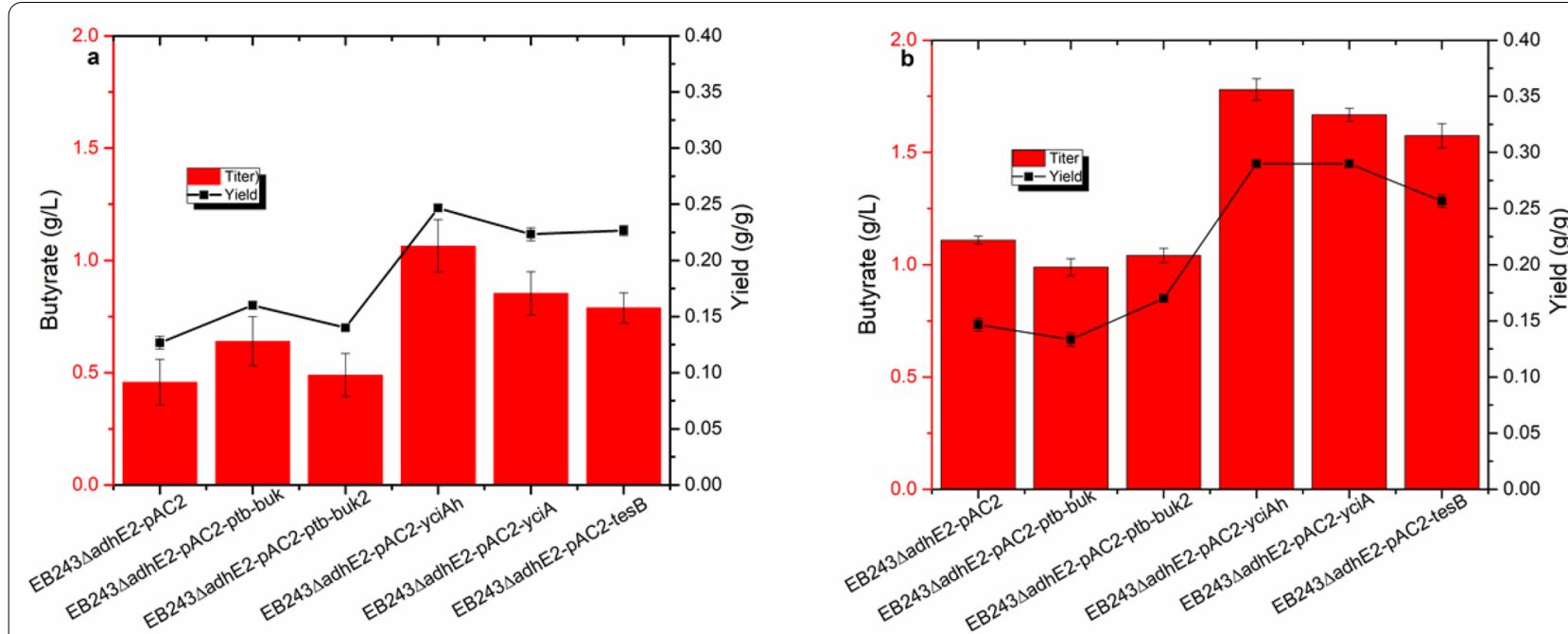

Fig. 2 Effects of overexpressing different butyrate-biosynthesis genes on the product titer. In a the strains were cultured in $10 \mathrm{~mL}$ polypropylene centrifuge tubes containing $5 \mathrm{~mL}$ of M9Y medium, while in $\mathbf{b}$ the strains were cultured in $50 \mathrm{~mL}$ polypropylene centrifuge tubes containing $5 \mathrm{~mL}$ of M9Y medium. The designation "yciAh" represents a synthesized version of the yciA gene from Haemophilus influenzae. The data represent the means \pm SD from three biological replicates 
synthesized yciAh gene from $H$. influenzae manifested the highest butyrate production of $1.06 \mathrm{~g} / \mathrm{L}$ in $72 \mathrm{~h}$, with a yield of $0.29 \mathrm{~g} / \mathrm{g}$ glucose, while the butyrate production of the strains containing all the other genes was below $1 \mathrm{~g} / \mathrm{L}$ (Fig. 2a).

The supply/consumption of NADH is balanced in strain EB243, which is capable of efficiently producing butanol. However, once strain EB243 was engineered from butanol production to butyrate production, the metabolic changes resulted in an excess of NADH. This is because the NADH consumed by aldehyde/alcohol dehydrogenase (encoded by adhE2) in strain EB243, cannot be recycled in the adhE2-deleted strain EB243 $\triangle$ adhE2, and the pathway for butyrate production does not require $\mathrm{NADH}$. To recycle the NADH and thus continue the fermentation, oxygen needs to be supplied for butyrate production. In fact, strain EB243 $\Delta$ adhE2 did not grow, nor produce butyrate, under anaerobic conditions (data not shown).

Subsequently, the production of butyrate by the six constructed strains in $50 \mathrm{~mL}$ polypropylene conical tubes containing $5 \mathrm{~mL}$ M9Y medium was quantified and compared. All strains produced a higher titer of butyrate (Fig. 2b) compared to the titer observed when the fermentation was performed in $10 \mathrm{~mL}$ polypropylene centrifuge tubes (Fig. 2a). Strain EB243 $\triangle$ adhE2-pAC2-yciAh produced the highest butyrate titer among all recombinant strains. After changing the culture volume, additional tube fermentation experiments were performed to study the effect of aeration on butyrate production. It was found that strain EB243 $\triangle$ adhE2-pAC2-yciAh produced a maximal butyrate titer of $3.5 \mathrm{~g} / \mathrm{L}$ with a yield of $0.34 \mathrm{~g} / \mathrm{g}$ glucose when $30 \mathrm{~mL}$ of M9Y medium was used in a $50 \mathrm{~mL}$ tube (Additional file 1: Fig. S1c). This suggests that moderate aeration is needed for butyrate production.

\section{Batch fermentation for butyrate production}

The yciAh gene, which resulted in the best butyrate production in tube-scale fermentations, was integrated chromosomally to obtain an antibiotic-independent fermentation strain. The resulting strain EB243ऽadhE2::yciAh was further subjected to bioreactor fermentation to evaluate its butyrate production ability. As the butyrate production is strongly related to oxygen availability, the fermentation system was aerated at rates of 0.3, 0.5, 0.75, and $1 \mathrm{vvm}$ (volume of gas per volume of liquid per minute) (Additional file 1: Fig. S2), which resulted in final $\mathrm{OD}_{600}$ values of $16.2,17.2,13.2$, and 13.9, respectively (Additional file 1: Fig. S2a). Strain EB243 $\triangle$ adhE2::yciAh produced $12.4 \mathrm{~g} / \mathrm{L}$ of butyrate under the aeration rate of $0.5 \mathrm{vvm}$. A promising butyrate yield of $0.46 \mathrm{~g} / \mathrm{g}$ of glucose ( $93.9 \%$ of the theoretical yield) and a productivity of $0.17 \mathrm{~g} / \mathrm{L} / \mathrm{h}$ were achieved after $72 \mathrm{~h}$, which was the highest yield reported for $E$. coli to date (Wang et al. 2019). A higher air flow of 0.75 or $1 \mathrm{vvm}$ did not favor butyrate production.

Normally, butyrate is produced by clostridia under anaerobic conditions through the ptb-buk pathway, while thioesterase genes are commonly found in aerobic microorganisms. Since aerobic conditions are required for the production of butyrate by engineered $E$. coli, the thioesterase may function better than $p t b$-buk under aerobic conditions. This is likely the reason why a single thioesterase out-performs $p t b$-buk for butyrate production. Although a certain amount of butyryl-CoA may be produced by fatty acid degradation (FAD) (Iram and Cronan 2006), it is questionable whether this contributed significantly to the produced butyrate. However, since it is inordinately challenging to calculate how much fatty acids are degraded to yield butyryl-CoA in a growing $E$. coli, and the fact that no external fatty acids were added to the medium, the quantity of butyrate that may have been derived from FAD was not evaluated in this study.

\section{Co-production of butanol and butyrate by the consortium} Using the butyrate-producing strain EB243AadhE2::yciAh and the butanol-producing strain EB243 (Dong et al. 2017), a microbial consortium was built to simultaneously produce the butanol and butyrate required for $\mathrm{BB}$ biosynthesis. However, the constructed consortium could not produce butyrate under anaerobic conditions, while under aerobic conditions, it could produce butyrate but the butanol production would be impaired. Considering the demand of moderate aeration for butyrate biosynthesis as described above, and the anaerobic conditions suitable for butanol fermentation (Dong et al. 2017), the mismatched oxygen demand would be a challenge for the synchronous production of butyrate and butanol. To address this challenge, the ratio of the butyrate- and butanol-producing strains was altered to enable the consortium to produce both butyrate and butanol under moderately aerobic conditions. The ratio of 1:4 (butyrate strain: butanol strain) was shown to be the best for the simultaneous production of butyrate and butanol in tube fermentation. Using this strategy, the titer of butyrate and butanol reached 2.5 and $2.4 \mathrm{~g} / \mathrm{L}$, respectively (Fig. 3 and Additional file 1: Fig. S3f). Notably, butanol production under aerobic conditions increased along with the increased ratio of the butanol-producing strain in the consortium, suggesting that altering the ratio of the consortium is an effective approach to optimize production. 


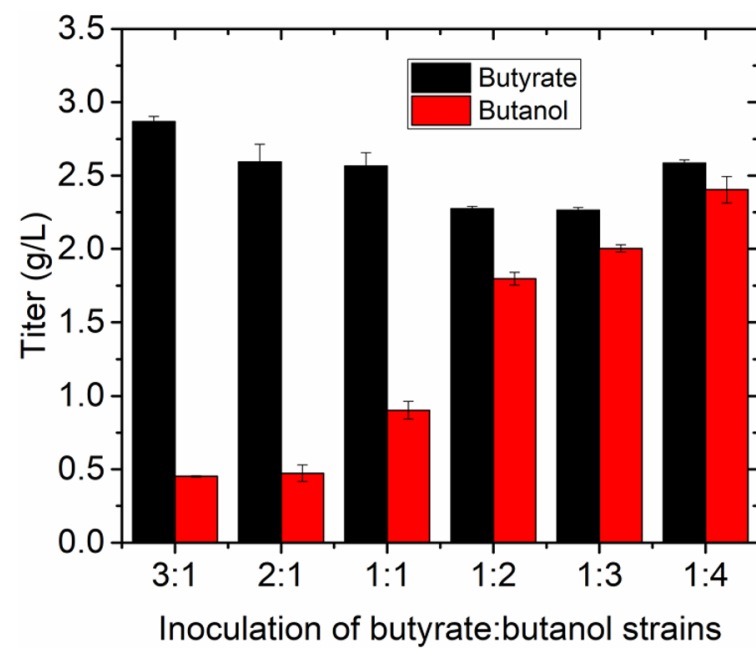

Fig. 3 Production of butyrate and butanol using consortia with varying inoculation ratios of the butyrate- and butanol-producing strains. The titers of butyrate and butanol were measured after $72 \mathrm{~h}$ of tube fermentation. The data represent the means \pm SD from three biological replicates

In situ production of BB using the microbial consortium in the presence of lipase Batch fermentation for the direct in situ production of $B B$

The production of $\mathrm{BB}$ by engineered $E$. coli is generally low (at $\mathrm{mg} / \mathrm{L}$ levels) even with the supplementation of exogenous substrates. In one study, alcohol acyltransferase (AAT) from Fragaria ananassa, a cultivated strawberry, was successfully expressed in E. coli, and the resulting strain subsequently cultured with exogenous $\mathrm{BB}$ precursors. However, only $0.28 \mathrm{mg} / \mathrm{L} \mathrm{BB}$ was produced when $1 \mathrm{~g} / \mathrm{L}$ butanol and $3 \mathrm{~g} / \mathrm{L}$ butyryl-CoA were added to the system (Horton and Bennett, 2006). In 2014, various modules related to the production of alcohols, along with an alcohol O-acyltransferase (ATF1) from S. cerevisiae, which is known to catalyze the last step of ester biosynthesis, were designed and introduced into E. coli. However, in spite of many esters produced in the mixture, no $\mathrm{BB}$ was detectable. Based on the idea that this strategy could provide butyryl-CoA, the process was supplemented with $3 \mathrm{~g} / \mathrm{L}$ of butanol, which resulted in a low $\mathrm{BB}$ titer of $14.9 \mathrm{mg} / \mathrm{L}$ (Rodriguez et al. 2014). Another study aimed to engineer $E$. coli to produce BB via fermentative biosynthesis (Layton and Trinh 2014). In said study, the enzymatic ester pathway with the AAT sub-module from Fragaria ananassa as introduced into E. coli to generate alcohol and acyl-CoAs molecules. However, no $\mathrm{BB}$ was detected among the produced esters, which may have been caused by insufficient butanol in the system. Although BB could finally be produced after adding $2 \mathrm{~g} / \mathrm{L}$ butanol, the titer of $36.8 \mathrm{mg} / \mathrm{L}$ was low. A further study demonstrated the prospect of constructing biotechnological carboxylate-to-ester platforms. To implement this, a modular E. coli chassis cell was precisely assembled using heterologous pathways comprising an acid to acylCoA synthesis sub-module (acyl-CoA transferase), an acyl CoA and alcohol condensation sub-module (alcohol acyltransferase), and an alcohol production sub-module. When the strain was fermented with glucose to form a combinatorial biosynthesis of fermentative esters, $2 \mathrm{~g} / \mathrm{L}$ butyrate was supplemented to the fermentation medium to reinforce the CoA molecule. However, only $47.6 \mathrm{mg} / \mathrm{L}$ BB was produced (Layton and Trinh 2016).

The reason for the low titers produced by these engineered $E$. coli strains is difficult to fully elucidate due to insufficient information on the characteristics of alcohol acyltransferase. Moreover, the biotechnological production of $\mathrm{BB}$ is also close linked with the supply of intrinsic precursors such as butyryl-CoA and butanol. Preferably, both substrates should be produced at a ratio of 1: 1 for efficient conversion of sugars into BB. However, this is a problem in E. coli, since butyryl-CoA and butanol are produced in a complex and interlinked metabolic pathway, making it challenging to balance their ratio.

To address this challenge, batch fermentation of a consortium comprising separate engineered butyrate- and butanol-producing strains was carried out in bioreactors with $0.5 \mathrm{~L}$ of modified M9 medium and $0.5 \mathrm{~L}$ hexadecane as an extractant, allowing the in situ removal of BB from the aqueous phase to avoid potential product inhibition. The $\mathrm{pH}$ was maintained at 6 , and air was sparged at a rate of $0.5 \mathrm{vvm}$ for aerobic growth. Additionally, an agitation speed of $200 \mathrm{rpm}$ was maintained, since thoroughly mixing the butyrate and butanol is essential for the lipasecatalyzed esterification reaction (Additional file 1: Fig. S4). Under these conditions, $1.1 \mathrm{~g} / \mathrm{L} \mathrm{BB}$ was produced at the end of the fermentation (Fig. 4c). While $7.1 \mathrm{~g} / \mathrm{L}$ butyrate was still present in the fermentation broth at this point, the residual butanol concentration was only $1 \mathrm{~g} / \mathrm{L}$ (Fig. 4d). Hence, insufficient butanol can explain the low BB titer. Additionally, as $\mathrm{pH}$ may affect the dissociation status of butyrate, the effect of $\mathrm{pH}$ was also further investigated.

\section{Optimization of $\mathrm{pH}$ control for improved BB production}

Butyrate is present in un-dissociated at low $\mathrm{pH}$, which may favor the esterification of butyrate with butanol to produce BB (Harroff et al. 2019; Zhang et al. 2017). However, low $\mathrm{pH}$ may severely impair bacterial cell growth and lead to poor fermentation performance (Maddox et al. 2000). Therefore, the effect of $\mathrm{pH}$ on the production of $\mathrm{BB}$ by the consortium in the bioreactor was investigated. 

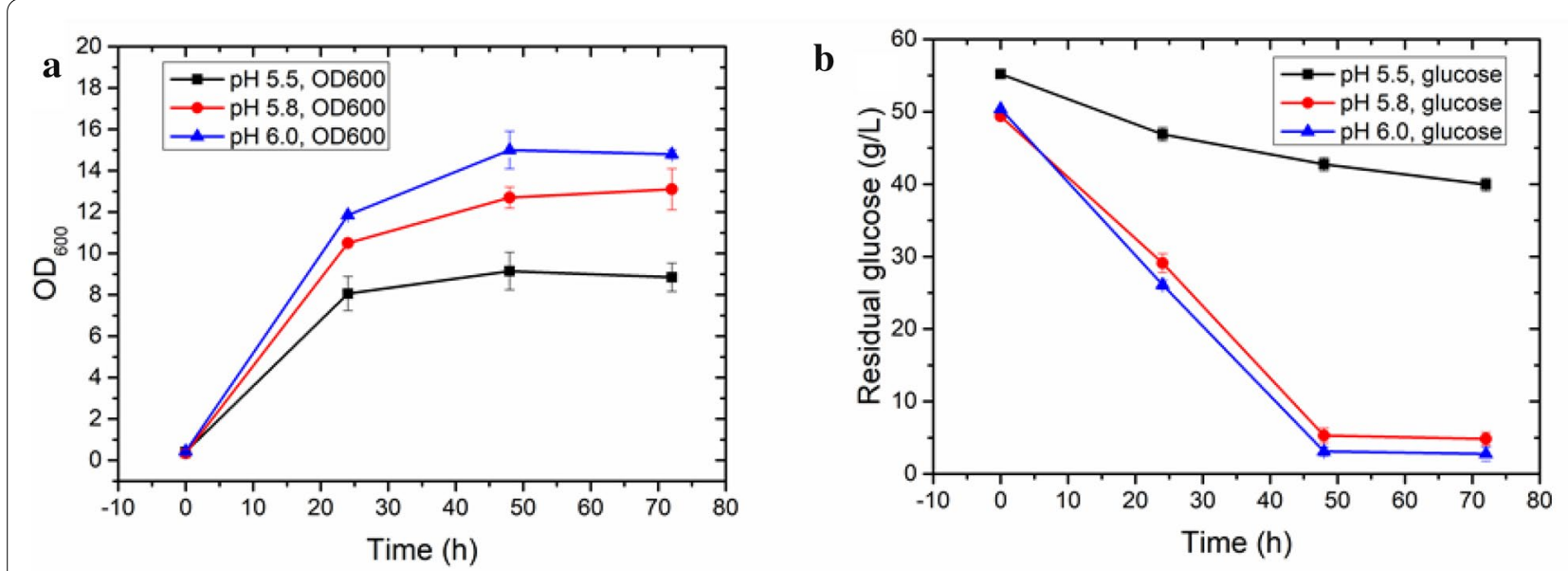

c

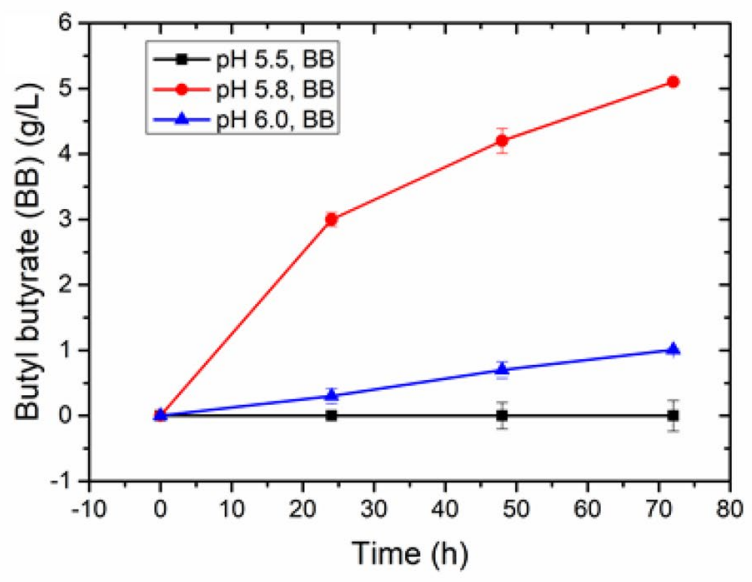

d

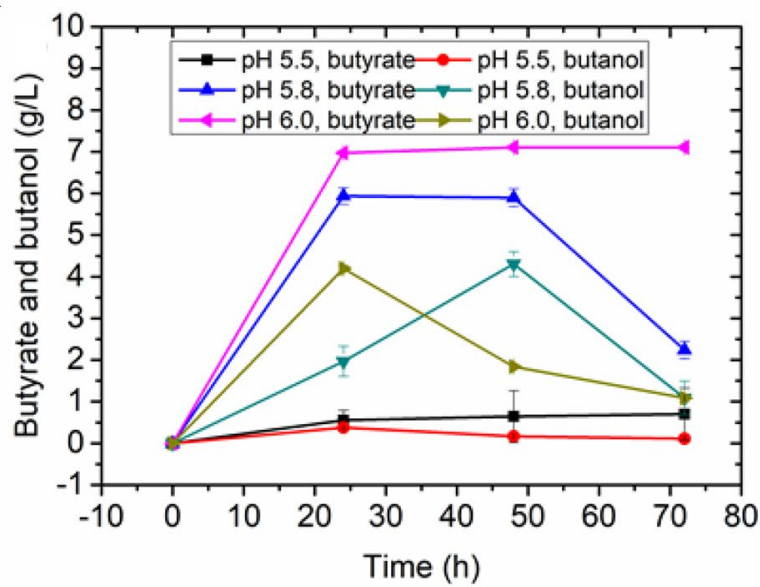

Fig. 4 Bioreactor batch fermentation profiles of the two strains for butyl butyrate production at different pH values. The strains were seeded at an

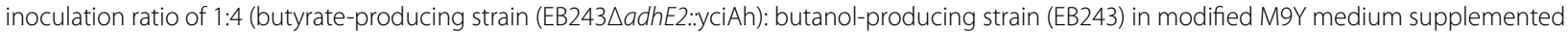
with $50 \mathrm{~g} / \mathrm{L}$ glucose. The system was composed of the fermentation broth and hexadecane as extractant at a volume ratio of 1:1. a Optical density at $600 \mathrm{~nm}\left(\mathrm{OD}_{600}\right)$, b glucose consumption, $\mathbf{c}$ butyl butyrate produced during the fermentation, and $\mathbf{d}$ butyrate and butanol. All parameters were assessed at pH 5.5, 5.8, and 6.0. Butyrate, $\mathrm{OD}_{600}$, butanol, butyl butyrate, and glucose were measured every $24 \mathrm{~h}$. Error bars indicate the standard deviations from three biological replicates

At $\mathrm{pH} 4.5$ and 5 , the consortium could not grow (data not shown), while at $\mathrm{pH} 5.5$, the microbial consortium grew moderately, but there neither butyrate nor butanol was detectable (Fig. 4d). This is closely related to the poor growth of the strains, leading to slow glucose utilization, which was reflected in the high residual concentration (Fig. 4b). This means that $\mathrm{pH}$ lower than 5.5 is not suitable for the simultaneous biosynthesis of butyrate and butanol in E. coli (Fig. 4). To tackle this challenge, a parallel experiment was performed at $\mathrm{pH}$ 5.8. At this $\mathrm{pH}$, the consortium was able to grow well (Fig. 4a), and almost all the glucose was consumed (Fig. 4b). Additionally, $2.2 \mathrm{~g} / \mathrm{L}$ butyrate along with $1.0 \mathrm{~g} / \mathrm{L}$ butanol remained in the fermentation medium at the end of fermentation. Overall, the consortium produced $5.1 \mathrm{~g} / \mathrm{L} \mathrm{BB}$ (Fig. 4c).

\section{Increasing the ratio of the butanol-producing strain favored BB production}

In the optimization experiments, the concentrations of butanol in the fermentation broth were always lower than the corresponding concentrations of butyrate. Therefore, BB synthesis was likely limited by the supply of butanol. Since the ratio of butanol to butyrate is the key factor determining the final production of $\mathrm{BB}$, it can be controlled by adjusting two process variables. One is the initial inoculation ratio of the butanol- and butyrateproducing strains, and the other is aeration, which affects both butanol and butyrate production. In tube fermentation, a ratio of 1:4 of the butyrate- and butanolproducing strains was shown to generate a comparable amount of butyrate and butanol. However, applying 
such a ratio in the fermenter left a final titer of $7.1 \mathrm{~g} / \mathrm{L}$ of unconsumed butyrate in the fermentation due to insufficient butanol. Since the conditions in the fermenter do not favor butanol production, the initial inoculum of the butanol-producing strain needed to be increased. As a consequence, increasing the ratio of the butyrate- and butanol-producing strains from 1:4 to 1:8 increased the $\mathrm{BB}$ production to $6.1 \mathrm{~g} / \mathrm{L}$. However, this was accompanied by a low yield of $0.09 \mathrm{~g} / \mathrm{g}$ glucose, whereby the residual concentrations of butyrate and butanol were roughly the same (Fig. 5b). Compared with the control consortium inoculated at a ratio of 1:4 (butyrate strain: butanol strain), it is evident that the consortium inoculated at a ratio of 1:8 grew significantly better (Fig. 5a). Once the inoculum ratio was optimized and fixed at the fermentor scale, the next key factor to be optimized was aeration. Since aeration favors butyrate production but inhibits butanol production, finding a balanced aeration strategy was challenging.

\section{Optimizing aeration to improve BB synthesis}

Since butyrate- and butanol-producing $E$. coli strains were required to construct a "diamond-shaped" consortium for efficient BB production, optimal aeration is necessary to produce a balanced ratio of the two precursors. When the consortium inoculated at a ratio of ratio $1: 4$ was grown under fully aerobic conditions, at $0.5 \mathrm{vvm}$, the butyrate titer reached $5.9 \mathrm{~g} / \mathrm{L}$ in $24 \mathrm{~h}$ of fermentation, while the butanol titer only reached $1.9 \mathrm{~g} / \mathrm{L}$ under the same conditions. By increasing the ratio from 1:4 to $1: 8$, the butanol titer reached $5.4 \mathrm{~g} / \mathrm{L}$, but there was only $1.6 \mathrm{~g} / \mathrm{L}$ of butyrate in the first $24 \mathrm{~h}$ of the fermentation.
This indicates that both strains in the consortium still need optimal aeration conditions to efficiently balance the butanol and butyrate supply, thereby increasing BB production. Since our previous research on butanol production indicated the need for microaerophilic conditions in the beginning of fermentation to facilitate cell growth, a two-stage aeration strategy for $\mathrm{BB}$ production was proposed. In this two-stage strategy, relatively high aeration is provided during the first stage to promote the growth of both butyrate- and butanol-producing strains, while also facilitating butyrate production. Subsequently, the aeration might be decreased at a certain time point to favor butanol production, while still sustaining butyrate production. Such a two-stage aeration strategy is expected to result in a better balance between the different oxygen demands of the butyrate- and butanol-producing strains, thus achieving higher BB production.

When aerobic conditions were applied in the first $24 \mathrm{~h}$ (strategy 1), the excess NADH could be oxidized, resulting in efficient butyrate production. Since the consortium requires butanol and the corresponding strain does not tolerate excess aeration, an anaerobic stage would favor the production of butanol. Nevertheless, the BB titer produced via this strategy was not significantly increased compared to an entirely aerobic process ( $6.35 \mathrm{vs.} 6.1 \mathrm{~g} / \mathrm{L}$ ). Similar to the glucose consumption of the consortium grown in the one-stage fermentation (Fig. 5a), the consortium grown at an air flow rate of $0.5 \mathrm{vvm}$ in the first $24 \mathrm{~h}$ (strategy 1 ) or $36 \mathrm{~h}$ (strategy 2 ), then shifted to 0.1 vvm in the second stage of the fermentation up to $72 \mathrm{~h}$, consumed almost all glucose by the end of the fermentation (Fig. 6a). As shown in Fig. 6d, the consortium grown
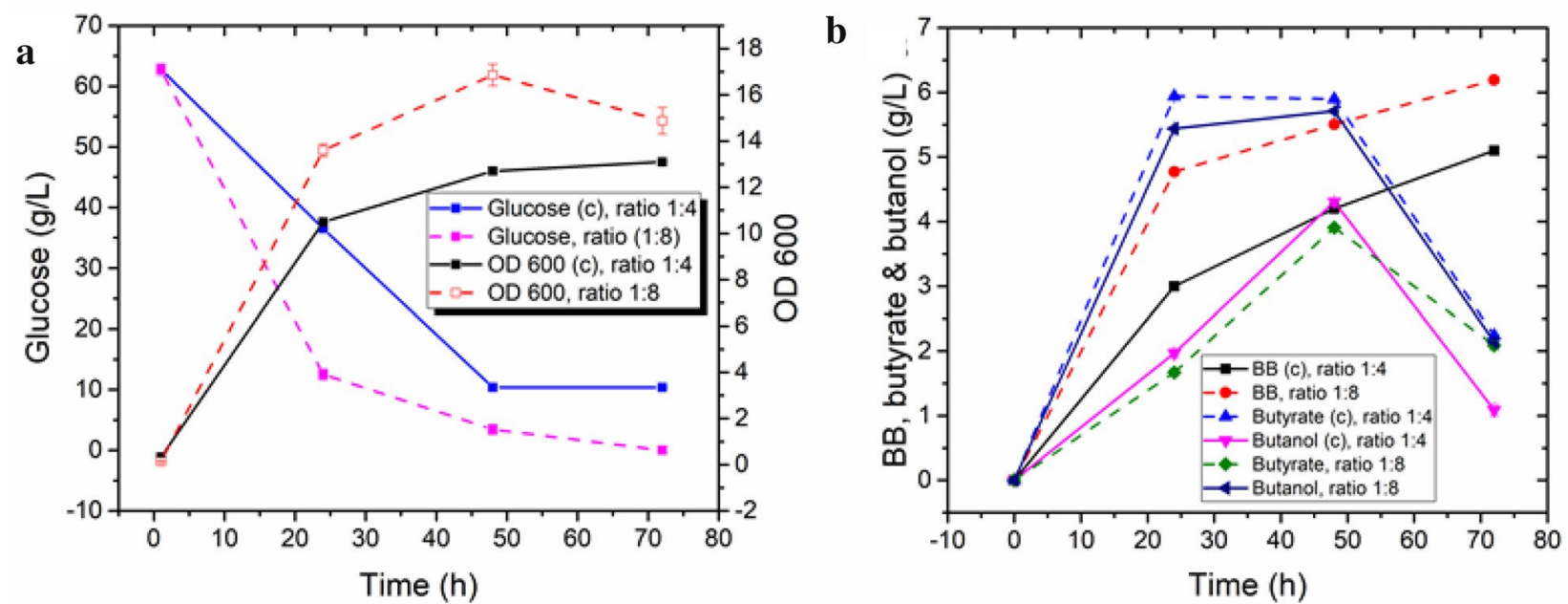

Fig. 5 Fermentation profiles of consortia with inoculation ratios (butyrate-producing strain: butanol-producing strain) of 1:4 (control, solid line) and 1:8 (dash line). a Profiles of optical density $\left(\mathrm{OD}_{600}\right)$ and glucose consumption. $\mathbf{b}$ Time-profiles of the butyrate, butanol, and butyl butyrate titers. The data represent the means \pm SD from three independent measurements 

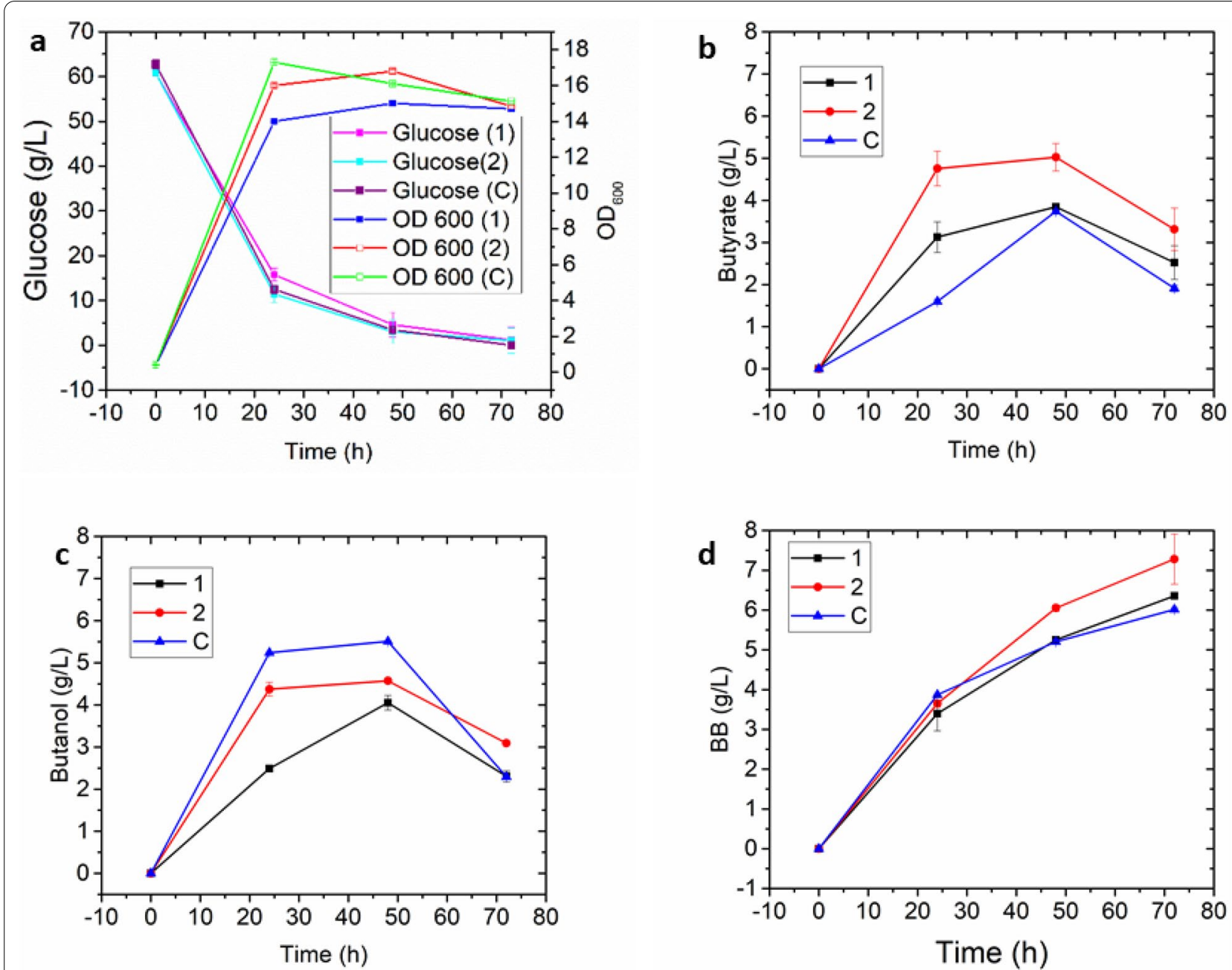

Fig. 6 Batch fermentation profiles of the microbial consortium for butyl butyrate production with different aeration speed control strategies. The consortium strains were inoculated at a ratio of 1:8 (butyrate strain: butanol strain) and cultured at $200 \mathrm{rpm}, \mathrm{pH}$ 5.8. C: control, aerated with $0.5 \mathrm{vvm}$ during the whole fermentation process. 1: aerated with $0.5 \mathrm{vvm}$ in the first $24 \mathrm{~h}$, then shifted to $0.1 \mathrm{vvm}$. 2: aerated with $0.5 \mathrm{vvm}$ in the first $36 \mathrm{~h}$, then shifted to $0.1 \mathrm{vvm}$. The data represent the means \pm SD from three biological replicates

using strategy 2 produced $7.2 \mathrm{~g} / \mathrm{L} \mathrm{BB}$ with a yield of $0.12 \mathrm{~g} / \mathrm{g}$ of glucose, compared to $6.35 \mathrm{~g} / \mathrm{L}$ BB with a yield of $0.11 \mathrm{~g} / \mathrm{g}$ of glucose in strategy 1 . Interestingly, the consortium grown using strategy 2 was still able to maintain the momentum to produce butanol (Fig. 6c) and butyrate (Fig. 6b), compared with the consortium without twostage aeration control. These results demonstrated that the approach not only increased the BB titer but also slightly increased the yield. However, the yield obtained is much lower than the theoretical yield of BB from glucose $(0.4 \mathrm{~g} / \mathrm{g})$. This yield limitation is most likely related to aeration, and needs further investigation beyond the scope of this study. As the ratio of initial inoculum and aeration both affect the production of butyrate and butanol, iterative optimization of these two factors is required to efficiently improve BB biosynthesis at a different scale of fermentation, as shown in this study.

Very recently, Cui et al. developed a microbial consortium to produce $\mathrm{BB}$. The consortium comprises two different species, C. beijerinckii BGS1 and C. tyrobutyricum ATCC25755, which could produce $6.8 \mathrm{~g} / \mathrm{L}$ butanol and $9.7 \mathrm{~g} / \mathrm{L}$ butyrate, respectively, during the co-culture fermentation process. After adding lipase, $5.1 \mathrm{~g} / \mathrm{L}$ of $\mathrm{BB}$ with a yield of $0.068 \mathrm{~g} / \mathrm{g}$ was produced. It is likely that the amount of butanol produced by $C$. beijerinckii BGS1 was insufficient for the consortium to achieve a high titer. Surprisingly, the BB yield of this Clostridium co-culture was 50\% lower than the yield achieved in our study $(0.12 \mathrm{~g} / \mathrm{g})$, although the Clostridium co-culture process is performed in an anaerobic process that does not require 
aeration and vigorous mixing. The low yield might be due to the accumulation of byproducts including acetone and isopropanol, whereas no significant byproducts were detected in our study. Another reason might be the imbalance between butanol and butyrate production in the Clostridium co-culture process, which hampers the further improvement of the titer and yield of BB. Moreover, since the Clostridium co-culture comprises two strains belonging to two different species, it is difficult, if not impossible, to synchronize the metabolism of these two Clostridium strains. By contrast, the two cognate $E$. coli strains in this study are almost identical except for a few genes. This makes it much easier to achieve homogeneity despite different inoculation ratios. Furthermore, E. coli fermentation does not require anaerobic handling and can therefore be more cost-effective.

The cognate consortium strategy used in this study corroborates very recent studies that demonstrated the ability of microbial consortia to relieve the metabolic burden of complex pathways when a single strain is used in biotransformations. By segregating biocatalytic pathway into three basic $E$. coli strains, hence constructing a cognate consortium, aliphatic $\alpha, \omega$-dicarboxylic acids (DCAs) (Wang et al. 2020) and 1,6-hexanediol (HDO) (Zhang et al. 2020) were produced at significantly higher rates than using a monoculture system.

\section{Conclusions}

In this study, we developed a cognate $E$. coli consortium for direct production of butyl butyrate from glucose in a one-pot process. The cognate $E$. coli consortium comprises a butyrate-producing strain and a butanol-producing strain, which share the same pathway upstream of butyryl-CoA. The nearly identical genotype of these two strains lightens the need for manipulation of nutritional conditions for this consortium. The cognate consortium was able to produce $7.2 \mathrm{~g} / \mathrm{L}$ butyl butyrate from glucose under suitable conditions without the exogenous addition of butanol or butyrate. This is the highest titer of butyl butyrate directly produced from glucose by $E$. coli reported to date, indicating the potential of using engineered E. coli consortia for the biotechnological production of esters. Although lipases are arguably the most widely used enzymes for the esterification of carboxylic acids with alcohols, their cost remains a problem, since an optimal balance between the output (titer, yield) and inputs (precursors, enzymes) is needed. To tackle this challenge, future studies should concentrate on overproducing recombinant lipases for selective ester biosynthesis. Moreover, the challenge of different oxygen requirements for butyrate and butanol biosynthesis needs to be addressed to achieve a higher yield of butyl butyrate.

\section{Supplementary Information}

The online version contains supplementary material available at https://doi. org/10.1186/s40643-021-00372-8.

Additional file 1. Additional figures and table.

\section{Abbreviations}

BB: Butyl butyrate; CoA: Coenzyme A; LCS: Recombinant lipase from Candida sp., expressed in Aspergillus niger; Novozymes Lipozyme ${ }^{\circledR}$ CALB; CRISPR:

Clustered Regularly Interspaced Short Palindromic Repeats; GC-MS: Gas chromatography-mass spectrometry; HPLC: High Performance Liquid Chromatography; NADH: Nicotinamide Adenine Dinucleotide; SD: Standard Deviation; OD: Optical Density; pH: Potential of Hydrogen; AAT: Alcohol Acyltransferase.

\section{Acknowledgements}

This research was supported by National Science Foundation of China (No. 31870038).

\section{Authors' contributions}

JPS, CZ, YL and YZ conceived and designed the study. JPS, CZ and GL performed the experiments. JPS analyzed the data. JPS, YL, and $Y Z$ wrote the paper. All authors read and approved the final manuscript.

\section{Funding}

This research was supported by National Science Foundation of China (No. 31870038).

\section{Availability of data and materials}

The supporting documentation showing the findings of this study is available from the corresponding author upon reasonable request.

Ethics approval and consent to participate

Not applicable.

Consent for publication

Not applicable.

\section{Competing interests}

The authors declare no conflict of interest.

\section{Author details}

${ }^{1}$ CAS Key Laboratory of Microbial Physiological and Metabolic Engineering, State Key Laboratory of Microbial Resources, Institute of Microbiology, Chinese Academy of Sciences, Beijing 100101, China. ${ }^{2}$ University of Chinese Academy of Sciences, Beijing 100049, China.

Received: 4 December 2020 Accepted: 15 February 2021

Published online: 21 February 2021

\footnotetext{
References

Chung H, Yoon MK, Han J, Kim Y-S (2015) Evaluation of volatile organic compounds in alcoholic beverages consumed in Korea. J Korean Soc Appl Biol Chem 58:423-432. https://doi.org/10.1007/s13765-015-0059-1

Cui Y, He J, Yang KL, Zhou K (2020) Production of isopropyl and butyl esters by Clostridium mono-culture and co-culture. J Ind Microbiol Biotechnol 47:543-550. https://doi.org/10.1007/s10295-020-02279-3

Dong H, Zhao C, Zhang T, Zhu H, Lin Z, Tao W, Zhang Y, Li Y (2017) A systematically chromosomally engineered Escherichia coli efficiently produces butanol. Metab Eng 44:284-292. https://doi.org/10.1016/j.ymben .2017.10.014

Harroff LA, Liotta JL, Wangolo EE, Egan TJS, Bowman DD, Angenent LT (2019) Field-scale co-fermentation of solid waste from urine-diverting dry toilets (UDDT-SW) and banana waste to produce undissociated carboxylic acids to inactivate ascaris eggs. Front Environ Sci 7:139. https://doi. org/10.3389/fenvs.2019.00139
} 
Horton CE, Bennett GN (2006) Ester production in E. coli and C. acetobutylicum. Enzyme Microb Technol 38:937-943. https://doi.org/10.1016/j.enzmi ctec.2005.08.025

Iram SH, Cronan JE (2006) The beta-oxidation systems of Escherichia coli and Salmonella enterica are not functionally equivalent. J Bacteriol 188:599_ 608. https://doi.org/10.1128/JB.188.2.599-608.2006

Jenkins RW, Munro M, Nash S, Chuck CJ (2013) Potential renewable oxygenated biofuels for the aviation and road transport sectors. Fuel 103:593599. https://doi.org/10.1016/j.fuel.2012.08.019

Jiang Y, Chen B, Duan C, Sun B, Yang J, Yanga S (2015) Multigene editing in the Escherichia coli genome using the CRISPR-Cas9 system. Appl Environ Microbiol 81:2506-2514. https://doi.org/10.1128/AEM.04023-14

Ju IB, Lim H-W, Jeon W, Suh DJ, Park M-J, Suh Y-W (2011) Kinetic study of catalytic esterification of butyric acid and n-butanol over Dowex 50Wx8-400. Chem Eng J 168:293-302. https://doi.org/10.1016/j.cej.2010.12.086

Kang SY, Park CH, Yoon YS, Cho IH, Ahn HW, Park SR, Song JH, Lee SH, Sang BI, Suh YW, Um YS, Lee SM (2011) Method of extracting butyric acid from fermented liquid and chemically converting butyric acid into biofuel. US Patent 8728782-B2, 20 May 2014.

Layton DS, Trinh CT (2014) Engineering modular ester fermentative pathways in Escherichia coli. Metab Eng 26:77-88. https://doi.org/10.1016/j.ymben .2014.09.006

Layton DS, Trinh CT (2016) Expanding the modular ester fermentative pathways for combinatorial biosynthesis of esters from volatile organic acids. Biotechnol Bioeng 9999:1-13. https://doi.org/10.1002/bit.25947

Maddox IS, Steiner E, Hirsch S, Wessner S, Gutierrez NA, Gapes JR, Schuster KC (2000) The cause of "acid crash" and "acidogenic fermentations" duping the batch acetone-butanol-ethanol (ABE-) fermentation process. J Mol Microbiol Biotechnol 2:95-100

Matte CR, Bordinhão C, Poppe JK, Rodrigues RC, Hertz PF, Ayub MAZ (2016) Synthesis of butyl butyrate in batch and continuous enzymatic reactors using Thermomyces lanuginosus lipase immobilized in Immobead 150. J Mol Catal, B Enzym 127:67-75. https://doi.org/10.1016/j.molca tb.2016.02.016

Menon N, Pasztor A, Menon BR, Kallio P, Fisher K, Akhtar MK, Leys D, Jones PR Scrutton NS (2015) A microbial platform for renewable propane synthesis based on a fermentative butanol pathway. Biotechnol Biofuels 8:61. https ://doi.org/10.1186/s13068-015-0231-1

Rodriguez GM, Tashiro Y, Atsumi S (2014) Expanding ester biosynthesis in Escherichia coli. Nat Chem Biol 10:259-265. https://doi.org/10.1038/ nchembio.1476
Santos JC, Nunes GFM, Moreira ABR, Perez VH, de Castro HF (2007) Characterization of Candida rugosa lipase immobilized on Poly(N-methylolacrylamide) and its application in butyl butyrate synthesis. Chem Eng Technol 30:1255-1261. https://doi.org/10.1002/ceat.200700153

Van den Berg C, Heeres AS, Van der Wielen LA, Straathof AJ (2013) Simultaneous Clostridial fermentation, lipase-catalyzed esterification, and ester extraction to enrich diesel with butyl butyrate. Biotechnol Bioeng 110:137. https://doi.org/10.1002/bit.24618

Wang L, Chauliac D, Moritz BE, Zhang G, Ingram LO, Shanmugam KT (2019) Metabolic engineering of Escherichia coli for the production of butyric acid at high titer and productivity. Biotechnol Biofuels 12:62. https://doi. org/10.1186/s13068-019-1408-9

Wang F, Zhao J, Li Q, Yang J, Li R, Min J, Yu X, Zheng GW, Yu HL, Zhai C, Acevedo-Rocha CG, Ma L, Li A (2020) One-pot biocatalytic route from cycloalkanes to alpha, omega-dicarboxylic acids by designed Escherichia coli consortia. Nat Commun 11:5035. https://doi.org/10.1038/s4146 7-020-18833-7

Xin F, Basu A, Yang KL, He J (2016) Strategies for production of butanol and butyl-butyrate through lipase-catalyzed esterification. Bioresour Technol 202:214-219. https://doi.org/10.1016/j.biortech.2015.11.068

Xin F, Zhang W, Jiang M (2019) Bioprocessing butanol into more valuable butyl butyrate. Trends Biotechnol 37:923-926. https://doi.org/10.1016/j.tibte ch.2019.03.012

Zhang ZT, Taylor S, Wang Y (2017) In situ esterification and extractive fermentation for butyl butyrate production with Clostridium tyrobutyricum. Biotechnol Bioeng 114:1428-1437. https://doi.org/10.1002/bit.26289

Zhang Z, Li Q, Wang F, Li R, Yu X, Kang L, Zhao J, Li A (2020) One-pot biosynthesis of 1,6-hexanediol from cyclohexane by de novo designed cascade biocatalysis. Green Chem 22:7476-7483. https://doi.org/10.1039/d0gc0 2600j

Zhao C, Sinumvayo JP, Zhang Y, Li Y (2019) Design and development of a "Y-shaped" microbial consortium capable of simultaneously utilizing biomass sugars for efficient production of butanol. Metab Eng 55:111-119. https://doi.org/10.1016/j.ymben.2019.06.012

\section{Publisher's Note}

Springer Nature remains neutral with regard to jurisdictional claims in published maps and institutional affiliations.

\section{Submit your manuscript to a SpringerOpen ${ }^{\circ}$ journal and benefit from:}

- Convenient online submission

- Rigorous peer review

- Open access: articles freely available online

- High visibility within the field

- Retaining the copyright to your article

Submit your next manuscript at $\boldsymbol{\nabla}$ springeropen.com 